\title{
La seguridad y protección portuaria. Un subespacio de políticas públicas de seguridad y gobernanza convergente
}

\author{
Security and Port Protection. A Subspace \\ of Public Security Policies and Converging Governance
}

Carlos Carmona Pérez

IPOLGOB-Universidad Carlos III de Madrid carlos.carmona@uc3m.es

\begin{abstract}
RESUMEN
Desde la última década del siglo $\mathrm{xx}$, las infraestructuras portuarias españolas catalogadas de interés general han sufrido una serie de trasformaciones políticas, económicas y sociales que arrojan como resultado un significativo cambio en los paradigmas clásicos de la gestión portuaria. En este sentido, la seguridad portuaria se ha convertido en uno de los aspectos de gestión más relevantes. Este artículo pretende, desde el análisis descriptivo, determinar los factores configuradores y dimensiones de un escenario singular de la acción pública, las políticas públicas de seguridad y protección portuaria. Dicha política pública tiene su especial significación en la actualidad, al emerger y consolidarse como un subespacio fuertemente característico de las políticas públicas de seguridad y que, a su vez, deriva de un ámbito de gestión postgubernamental de gobernanza convergente.
\end{abstract}

\section{PALABRAS CLAVE}

Políticas públicas de seguridad y protección portuaria, políticas públicas, gobernanza, actores, Administración portuaria.

\begin{abstract}
Since the last decade of the twentieth century, the Spanish port infrastructures cataloged of general interest has undergone a series of political, economic and social transformations that result in a significant change in the classic paradigms of port management. In this sense, port security has become one of the most relevant aspects of management. This article aims, from the descriptive analysis, to determine the configurator factors and dimensions of a singular scenario of public action, public security policies and port protection. This public policy has its special significance today, as it emerges and consolidates as a subspace that is strongly characteristic of public security policies and which, in turn, derives from a scope of post-governmental management of converging governance.
\end{abstract}

\section{KEYWORDS}

Public security policies and port protection, public policies, governance, actors, port Administration.

\section{SUMARIO}

1. INTRODUCCIÓN. 2. EL COMPLEJO MARCO DE LAS POLÍTICAS PÚBLICAS DE SEGURIDAD Y PROTECCIÓN PORTUARIA. 3. LA SEGURIDAD Y PROTECCIÓN PORTUARIA COMO SUBESPACIO CARACTERISTTICO DE LAS POLÍTICAS PÚBLICAS DE SEGURIDAD. 4. LAS POLÍTICAS PÚBLICAS DE SEGURIDAD Y PROTECCIÓN PORTUARIA EN ESCENARIOS DE GOBERNANZA CONVERGENTE. 5. CONCLUSIONES. 6. REFERENCIAS BIBLIOGRÁFICAS Y DOCUMENTALES. 


\section{INTRODUCCIÓN}

Las infraestructuras portuarias españolas catalogadas de interés general ${ }^{1}$ se encuentran inmersas en un constante desafío en lo que a la seguridad concierne, al generarse en ellas escenarios de acción institucional complejos e indeterminados que responden a un conjunto de factores y mecanismos interdependientes de actuación gubernamental como son la estructura, los procesos y el ámbito de decisión compartido con diferentes actores estatales y transnacionales.

Asimismo, no puede obviarse que la implementación de los modelos de gestión de las políticas públicas contemporáneas lleva aparejado un significativo cambio paradigmático derivado de aquello que identificamos como gobernanza de la acción pública (Vanaclocha, 2013; Wallace, 2000; Kassim, 2001); máxime si a esta la concebimos desde una perspectiva convergente, al quedar estrechamente influenciado por el entramado normativo internacional, de obligado cumplimiento para las instituciones político-administrativas de cada Estado, el marco de interacción reticular entre actores multinivel, de carácter público y privado, que comparten objetivos comunes y capacidad de decisión en el curso de acción pública de este tipo de políticas.

Desde una perspectiva empírica, este hecho incide de forma directa en el ámbito de la seguridad y protección portuaria, ya que, al observarse como un subespacio característico de las políticas públicas de seguridad, fomenta la configuración instrumental y estratégica en su ciclo de análisis.

No obstante, existe una diversidad de razones que permite explicar este fenómeno, partiendo de la transposición y posterior desarrollo en el entramado institucional de las normas europeas e internacionales en materia de seguridad portuaria (Dezalay y Garth, 2002), hasta culminar con los retos e impactos que supone la propia globalización de los actos ilícitos y terroristas en las infraestructuras portuarias (Drezner, 2005). Por ello, en este tipo de políticas públicas, los diferentes actores institucionales que participan en el proceso reorganizan e incorporan diferentes mecanismos y estrategias de intervención, provenientes del marco regulador transnacional, pero que, en todo caso, se conjugan con el sistema de valores y la cultura organizativa nacional (Fligstein, 2008; Knill y Lehmkuhl, 2002).

De este modo, puede percibirse una cierta mimetización fundamentada en la propia globalización instrumental, al estimular a los actores para que asuman mecanismos que «funcionan» en otros países (Powell y DiMaggio, 1991), ya que la interdependencia intrínseca de ésta genera compartir retos y respuestas homogéneas en el ámbito de la seguridad pública.

Por lo tanto, partiendo de que las políticas públicas de seguridad en las infraestructuras portuarias españolas implican la combinación geométrica de distintas variables, de la estandarización instrumental y del marco de interacción con el entorno, el presente artículo pretende analizar aquellos factores configuradores y rasgos significativos que permiten identificar a las políticas de seguridad y protección portuaria como subespacio característico de las políticas públicas de seguridad y de gobernanza convergente en los puertos españoles de interés general. Perspectiva esta de especial significación, al construirse desde la óptica del análisis de los procesos, de la toma de decisiones y de la gestión de sus resultados e impactos. Para el desarrollo de este estudio se han utilizado diversos informes de gestión y fuentes de información cuantitativa y cualitativa suministradas por el ente público Puertos del Estado.

\section{EL COMPLEJO MARCO DE LAS POLÍTICAS PÚBLICAS DE SEGURIDAD Y PROTECCIÓN PORTUARIA}

La implementación explícita de las políticas públicas de seguridad y protección portuaria deriva del cambio conceptual de la seguridad colectiva, que tiene su razón de ser en las transformaciones políticas, económicas y sociales producidas en las sociedades democráticas avanzadas a partir de la última década del siglo Xx. Si tomamos como cierto que dichas transformaciones afectan de un modo significativo a todos los órdenes sociales, no es menos cierto que en ámbito específico de la seguridad tienen transcendentales consecuencias: la percepción de amenazas y niveles de riesgo por parte de los ciudadanos y la sociedad

Se entiende como puertos de interés general, o de titularidad estatal, a aquellos que por su circunstancia reúne, al menos, alguna de las siguientes características: se efectúan en ellos actividades marítimas internaciones; sirven industrias de importancia estratégica para la economía nacional; el volumen anual y las características de sus actividades comerciales marítimas alcanzan niveles suficientemente relevantes, o responden a necesidades esenciales de la actividad económica general del Estado, y sus especiales condiciones técnicas o geográficas son esenciales para la seguridad del tráfico marítimo nacional e internacional. 
civil, que en numerosas ocasiones sobrepasan la capacidad de respuesta de cada Estado (Velázquez, 2009:17), precisa la adaptación del ciclo de acción pública a un ámbito de demandas particular y determinado que permita la adecuación de los medios y procedimientos a un contexto cambiante y de difícil predicción.

Así, el cambio de la concepción ciudadana de seguridad individual y colectiva lleva aparejado un nuevo orden respecto a los paradigmas clásicos de diseño e implementación de las políticas públicas objeto de estudio, al concentrarse en ellas la acción, o inacción, gubernamental para proporcionar respuestas de carácter sectorial y transversal, mediante instrumentos y estructuras singulares, frente a las amenazas y riesgos que indicen sobre el conjunto de las infraestructuras portuarias (Vanaclocha, 2013: 4; Carmona, 2014).

Esta aproximación a la noción de políticas públicas de seguridad y protección portuaria permite suponer que el actual escenario de acción pública se configura de manera compleja e indeterminada, ya que además de la importancia que los resultados e impactos de su ejercicio representa para el entorno, el análisis de las demandas y apoyos es otro de los factores que ha mostrado también una cierta versatilidad.

En este sentido, los postulados emanados de los estudios sobre la sociedad del riesgo (Beck, 1992; Giddens, 1990) permiten describir de modo estructurado una esfera de desafíos gubernamentales en el ámbito de la seguridad portuaria, al tener como finalidad que los decisores públicos puedan ofrecer respuestas de carácter anticipativo a los distintos riesgos y amenazas derivadas de los actos ilícitos, el terrorismo internacional, los ciberataques, los accidentes intencionados o fortuitos, los conflictos sociales, o los desastres naturales.

Sin embargo, esta tarea en principio parece ardua, ya que la anticipación de acontecimientos futuros y la precognición en el diseño de las políticas de seguridad precisa soslayar ciertos obstáculos epistemológicos, como los modelos de gestión previos, y la implementación de técnicas de gestión que faciliten la toma de decisiones en el referido ámbito de la seguridad portuaria. Ello implica la importancia, aunque, con matices, que presenta el análisis, gradación y gestión de riesgos, al permitir realizar pronósticos de vulnerabilidad e impacto en términos generales, pero que, en todo caso, no son exactos (Losada, 2005: 27-50).

Por otro lado, la irrupción de la seguridad y protección portuaria como marco de análisis de las políticas públicas de seguridad se encuentra estrechamente ligada con las distintas transformaciones producidas en el espacio supranacional, así como en la globalización de las vulnerabilidades que en la actualidad afectan a las sociedades democráticas. Con todo, debe tenerse presente que como se ha podido argumentar con anterioridad es a partir de la última década del siglo xx cuando comienza a considerarse que la seguridad en su conjunto, y específicamente la seguridad portuaria, no escapa de la clásica concepción y análisis de las políticas públicas, al emerger y consolidarse con la función garantista de preservación de los derechos y libertades, así como del normal desarrollo social e institucional de una comunidad política. Dicha función, a su vez, genera un escenario de acción pública en el que se superponen distintas opciones, en principio, ambivalentes, al conjugar rasgos limitativos y garantistas en lo referente a los derechos y libertades públicas de los ciudadanos. Pero que se transforman en una concatenación de valores, ideas y paradigmas que influyen, en mayor o menor medida, en la agenda social y gubernamental -las arenas de decisión pública- a través del proceso de interacción que se produce entre los actores intervinientes.

En este sentido, puede deducirse que las políticas de seguridad y protección portuaria concentran la amalgama de la propuesta racional de regulación pública del conjunto de apoyos, demandas y desafíos que afrontan en la actualidad las infraestructuras portuarias españolas objeto de estudio. Sin embargo, dichas políticas no escapan de la concepción clásica que las percibe como un mecanismo de asignación pública de recursos y oportunidades entre los distintos actores sociales con intereses, preferencias y demandas en el "hecho regulador» (Subirats y Gomá, 2001: 561). Asimismo, y dado su alto componente institucional, estas responden a un proceso determinado y diferenciado de acción -o inacción-gubernamental que, mediante microcircuitos que concentran elementos de legitimación -o deslegitimación-, se orienta la configuración de un espacio explícito de problemas, demandas, necesidades y oportunidades (Aguilar Villanueva, 1992: 2140; Meny y Thoening, 1992; Subirats, 1989: 40-41).

Ahora bien, debe reconocerse que dada su marcada especificidad, las políticas de seguridad y protección portuaria responden a una categoría de análisis compleja que permite observarlas como un escenario de acción gubernamental característico e indeterminado, que comporta un ámbito de especial significación dentro de la actual estrategia española de seguridad nacional. Así, su análisis muestra que se trata de políticas de carácter transversal que comparten espacios secantes de acción pública con otro tipo de políticas sectoriales, ya que de un modo u otro generan resultados e impactos sociales y políticos relevantes, sean estos directos o indirectos. Igualmente, son políticas de carácter multiactoral, al implicar su ciclo de acción la participación 
de una pluralidad de actores políticos, institucionales y sociales; máxime cuando nos referimos a modelos de Estado con un elevado grado de desconcentración y descentralización político-administrativa. Y, por último, se trata de políticas de carácter compuesto porque implican escenarios de acción con dinámicas diversas y características complejas, así como, y a diferencia de otro tipo de política pública, la posibilidad de ejercer el uso legítimo de la fuerza como modo de acción pública del entramado institucional del Estado.

Se trata, pues, de políticas públicas que de un modo transversal se enmarcan en escenarios sensibles y estratégicos de la acción gubernamental, implicando la combinación de tres aspectos fundamentales que, aunque en principio puedan suponerse ambivalentes, no son más que los elementos de articulación de las dimensiones de las políticas públicas de seguridad en un sentido genérico: la autoridad gubernamental componente legitimador-, la coerción y la garantía de los derechos y libertades básicas de los ciudadanos.

Otro de los elementos que permite, no obstante, determinar la complejidad del marco de acción de las políticas de seguridad y protección portuaria contemporáneas es el relativo a la asunción de nuevos retos y estrategias contrapuestas al lenguaje burocrático clásico, al aproximarse a los postulados emanados del management público (ámbito de gestión postburocrático) y de la gobernanza (ámbito de gestión postgubernamental).

La gestión pública, así como el análisis de las políticas públicas de seguridad y protección portuaria se configuran, de este modo, como ámbitos de gestión secantes en las administraciones portuarias contemporáneas, ya sea en sus niveles de actuación macro o micro (Metcalfe, 1993: 181), al configurarse el ápice estratégico como la respuesta a las demandas y preferencias de los actores políticos, institucionales y sociales concurrentes en el escenario de acción propuesto. Por lo cual, debe entenderse que estas políticas se presentan en forma de variable de la gestión estratégica, al conformar el nexo de unión entre el sistema de valores y el proceso de implementación y evaluación de los resultados e impactos generados (Gomá y Subirats, 1999: 32).

Así pues, el diseño, la implementación y la evaluación de las políticas públicas de seguridad y protección portuaria, conjugado con la ejecución y gestión de los medios y medidas para la consecución de objetivos predefinidos, suponen el desarrollo de un conjunto basal de acciones y estrategias vinculantes. Pero que, en todo caso, responde a tres ámbitos de acción cuasi-secantes: análisis y diagnóstico del entorno portuario donde se focaliza la acción pública, que permite generar acervo de conocimiento de las demandas, de las oportunidades y de las amenazas, sean estas de carácter objetivo o subjetivo; análisis y diagnóstico de la organización, implicando el conocimiento de las fortalezas y debilidades que presenta la organización durante el proceso de desarrollo estratégico, y el planteamiento estratégico articulado mediante objetivos y acciones concordantes con la misión, la visión y los valores de la organización, así como con los retos identificados en el análisis y diagnóstico del entorno y la organización.

FiguRA 1. ACCIONES BASALES de LAS POLÍticAS PÚBLICAS DE SEGURIDAD

\begin{tabular}{l|l}
\hline \multirow{2}{*}{ Análisis entorno portuario } & Diagnóstico del entorno. \\
\cline { 2 - 2 } & Dimensión analítica de indicadores delincuenciales. \\
\hline \multirow{2}{*}{ Análisis organización portuaria } & Diagnóstico organizativo. \\
\cline { 2 - 2 } & Acciones de reforma y modernización administrativa. \\
\hline \multirow{4}{*}{ Planeamiento estratégico } & Misión, visión y valores. \\
\cline { 2 - 2 } & Líneas, objetivos y acciones centrífugas. \\
\cline { 2 - 2 } & Líneas, objetivos y acciones centrípetas. \\
\cline { 2 - 2 } & Evaluación de resultados e impactos. \\
\hline
\end{tabular}

Fuente: Elaboración propia

En definitiva, la combinación geométrica de cada uno de los elementos configuradores propuestos es la que permite percibir la complejidad del escenario de las políticas públicas de seguridad y protección portuaria, al depender su éxito, o fracaso, de diferentes factores estructurales, así como de los mecanismos de coordinación y cooperación institucional, que condicionan sobremanera este tipo de política pública de 
carácter esencialmente regulativo (Lowi, 1972; Gomá y Subirats, 1999: 24). En este sentido, las dinámicas provenientes de la gobernanza pública podrían suponerse como las variables que permitan facilitar y adaptar el diseño estratégico a la acción gubernamental.

Vistas las especificidades que emanan del marco anteriormente descrito, se analizará a continuación la dimensión de la seguridad y protección portuaria como subespacio de las políticas públicas de seguridad y gobernanza convergente.

\section{LA SEGURIDAD Y PROTECCIÓN PORTUARIA COMO SUBESPACIO CARACTERÍSTICO DE LAS POLÍTICAS PÚBLICAS DE SEGURIDAD}

Desde un enfoque sistémico, un rasgo esencial de la seguridad y protección portuaria es que constituye, en términos de análisis, un subespacio integrado en una política pública de mayor envergadura y alcance como es la seguridad en un sentido genérico. Pues, en ella se concentra, a nivel macro, una serie de procesos de acción de unidades y redes gubernamentales, pero también, a nivel micro, un conjunto de planes y estrategias que desarrollan escenarios específicos de acción gubernamental.

Se trata, por tanto, de un subespacio sensible y de gran valor estratégico estatal en el que se conjuga una frecuente transversalidad (apoyo a otros subespacios concentrados en las políticas públicas de seguridad) y un alto grado de conectividad con otros espacios de políticas públicas (como por ejemplo los de justicia o los del ámbito socioeconómico).

Ante este contexto parece lógico pensar que la identificación analítica y política del subespacio de la seguridad y protección portuaria resultó una nueva «ventana de oportunidad» (Kingdom, 1995), en cierto modo preferente, de las políticas públicas de seguridad, máxime si en él se concentra una perspectiva integradora de gobernanza convergente.

Sin embargo, debe reconocerse que dicho subespacio, entendido como la expresión político-administrativa de la acción de prestación pública de la seguridad en los entornos portuarios, es el escenario principal de las múltiples intervenciones que lleva a cabo la administración portuaria estatal. Su relevancia se manifiesta, en términos cuantitativos, por el significativo volumen de recursos económicos y humanos, y, desde una perspectiva cualitativa, en la configuración como un servicio básico de prestación directa que garantiza el normal funcionamiento de las infraestructuras portuarias tomadas como marco de referencia en este estudio.

En este sentido, la figura 2 muestra la evolución de las inversiones, durante el periodo 2009-2014, destinadas a la mejora de la seguridad y protección portuaria en el conjunto de puertos de interés general, tanto en valores absolutos como relativos en proporción con las inversiones totales.

FIGURA 2. EVOLUCIÓN DE LAS INVERSIONES EN MATERIA DE SEGURIDAD PORTUARIA

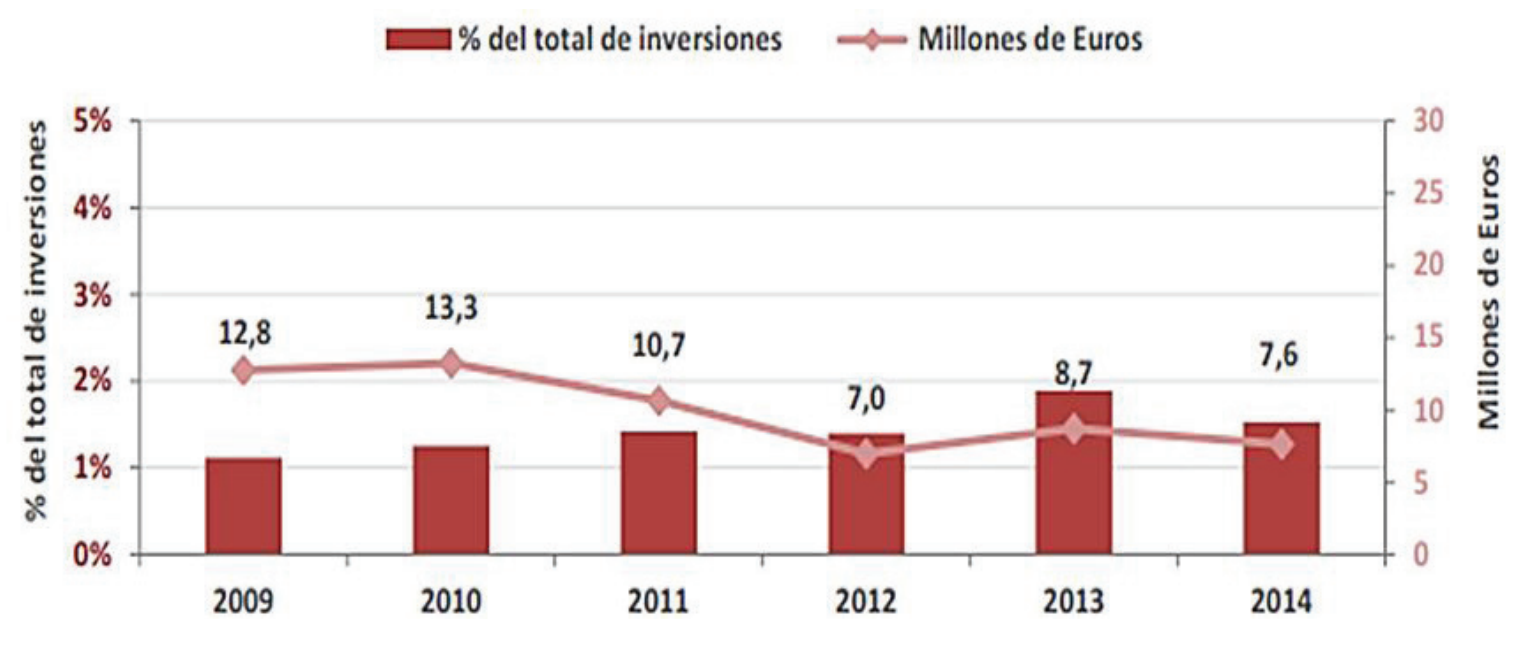

Fuente: Puertos del Estado

Asimismo, la figura 3 muestra la distribución de los recursos humanos por áreas de actividad en las infraestructuras que integran el sistema portuario de interés general. En ella puede observarse cómo la es- 
tructura de la plantilla está fuertemente condicionada por el personal de seguridad (policía portuaria), lo cual motiva que, en un $53,6 \%$ de las Autoridades portuarias, el mayor porcentaje analizado corresponda a dicho personal.

FIGURA 3. DISTRIBUCIÓN DE PLANTILLA AUTORIDADES PORTUARIAS POR ÁREAS DE ACTIVIDAD
口oficina fuera de convenio
Q Oficina dentro convenio
QMantenimiento
Q Policia Portuaria

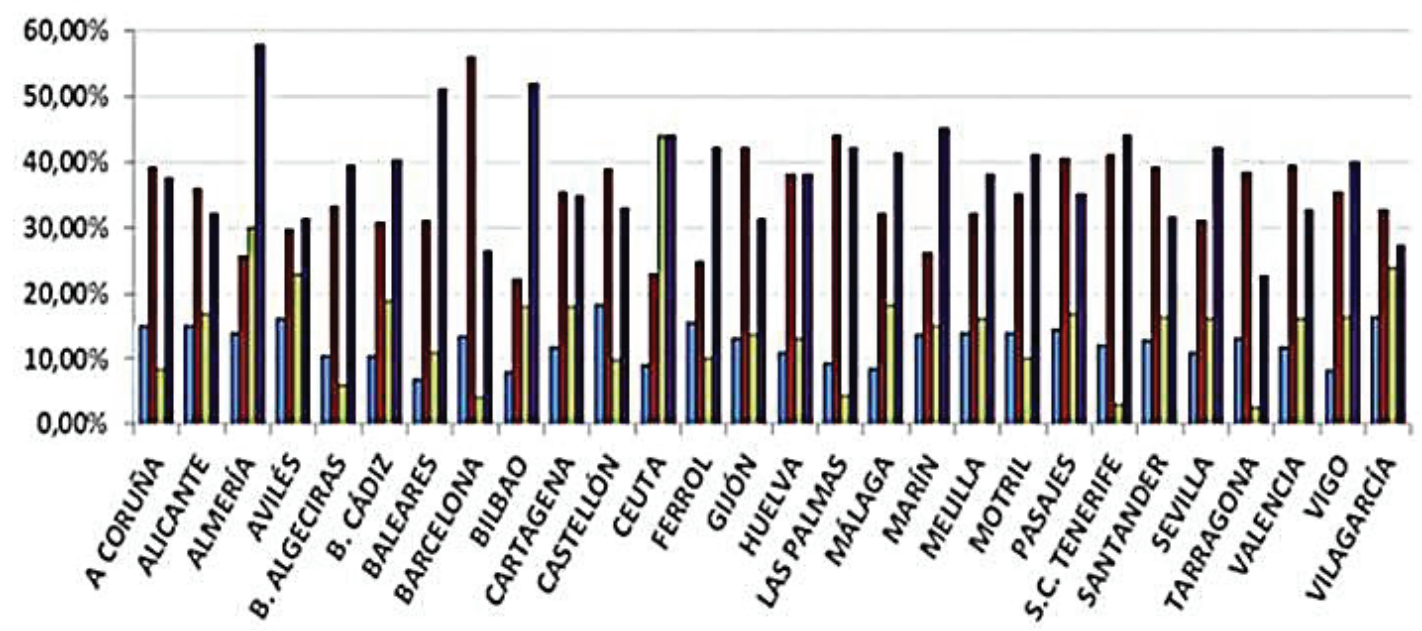

Fuente: Puertos del Estado

En consecuencia, el elemento central de incidencia de las políticas de seguridad y protección portuaria queda delimitado por las dimensiones del propio sistema en el que tiene lugar la acción pública. El ámbito portuario entendido como Administración instrumental diferenciada.

La concepción clásica del puerto como infraestructura de transporte en las sociedades desarrolladas ha sido objeto de profundas revisiones a partir de la segunda mitad del siglo XX. Tal circunstancia conllevaría a un nuevo planteamiento en lo concerniente al orden social y económico de la esfera marítimo-portuaria. No obstante, cabe recordar que dichas transformaciones tienen una dimensión universal, al sobrepasar las fronteras clásicas de los Estados, y acelerada: la interacción puerto-ciudad ha sido uno de los elementos vertebradores de esta nueva concepción, ya que surge a raíz de un nuevo escenario de conflicto entre la Administración portuaria y la Administración local que gestionaban hasta ese momento cada uno de los espacios competenciales definidos de modo no recíproco ${ }^{2}$.

Además, la evolución de la misión comercial de los puertos es considerada como otro de los elementos diferenciadores del orden social y económico portuario. Ello ha supuesto un cambio de los paradigmas clásicos de gestión portuaria, permitiendo el diseño de mecanismos de interacción ad hoc entre el puerto y la ciudad, eso es, que los puertos se han transformado en centros logísticos de la cadena de transporte, integrando su actividad con la sociedad.

El análisis del sistema portuario que nos ocupa permite determinar que este hecho tiene una especial significación, ya que en el espacio marítimo-portuario las competencias en materia de policía, seguridad y protección se presentan de modo concurrente debido a los roles a desempeñar por cada uno de los actores policiales en él presentes (Guardia Civil, Cuerpo Nacional de Policía, Policía Portuaria y Agentes de Vigilancia Aduanera, esencialmente).

Por ello, el desarrollo del actual escenario de gestión de las infraestructuras portuarias se ha mostrado proclive al reciente impulso del subespacio comprendido por la seguridad y protección portuaria, subespacio intrínseco al espacio de las políticas públicas de seguridad. La identificación del mismo implica el rediseño del anterior marco de acción pública, al precisar de una específica regulación, así como de la implementa-

2 Desde una perspectiva general, es preciso señalar que la identificación de las infraestructuras portuarias como espacios de centralidad urbana genera un primer escenario de conflicto puerto-ciudad, al poner de manifiesto las importantes plusvalías que esta condición genera. Asimismo, la barrera puerto-ciudad es otro relevante foco de conflicto entre las citadas administraciones, al resultar un obstáculo para el diseño de los planes municipales de transformación urbana. 
ción y evaluación de políticas, planes y programas que conciban un sistema plausible de seguridad y protección de las infraestructuras portuarias, sea este tanto de carácter preventivo como reactivo.

Sin embargo, ha de tenerse en cuenta la existencia de una serie de circunstancias relevantes en el instante de abordar el análisis de las políticas de seguridad y protección portuaria. Así, la categorización de estas como subespacio de las políticas públicas de seguridad guarda una estrecha relación con el fenómeno de que la inseguridad se traduce en problematización en términos políticos; hecho que servirá de estímulo a los poderes públicos, al facilitar la identificación de respuestas -las políticas- a las demandas (Tamayo, 1997: 296-298) en el ámbito de la seguridad y protección portuaria.

Por otro lado, el análisis de las respuestas gubernamentales y de la acción pública que tiene lugar en el escenario delimitado por la seguridad y protección de las infraestructuras portuarias, permite intuir que las mismas no siempre han sido formuladas de un modo simétrico. Este tipo de política pública en cuestión, al igual que sucede con cualquier otro tipo de política pública, ha adaptado tanto su oferta como su demanda a las necesidades cambiantes de su entorno. Significativamente, tal circunstancia ha supuesto una evolución desde parámetros de acción reactivos frente a los actos ilícitos, hacia parámetros globales de gestión preventiva de los riesgos y amenazas.

Ello supone precisar la identificación de complejos y novedosos escenarios de gestión supranacional en los que se imbrican las políticas de seguridad y protección portuaria. Pero que, en todo caso, sustentan su formulación como un claro ejemplo de pragmatismo político, al estar motivado su diseño incremental en otro espacio de política pública experimentado y, por tanto, previsible como es el de la defensa (Talavera, 2014: 29).

Asimismo, desde la perspectiva de análisis de políticas públicas, el subespacio de las políticas de seguridad y protección portuaria presenta una manifiesta reformulación de los planteamientos clásicos de gestión y organización de los actores institucionales partícipes en el ciclo de acción pública, ya que su origen parte de los procesos, eso es, que la política pública se presenta como la variable independiente del análisis y, por ello, se perfila como el elemento orientador de dichos actores respecto a su diseño, alcance, funciones y límites competenciales, así como del marco de interacción con el entorno.

Sobre la base de las reflexiones analíticas planteadas hasta el momento, es preciso señalar que este tipo de políticas públicas se instrumentan y configuran a partir de una serie de rasgos significativos como los que se describen a continuación.

a) El diseño y la implementación de las políticas públicas de seguridad y protección portuaria parte y se consolida en paralelo a la iniciativa supranacional de prevenir las vulnerabilidades y riesgos en las infraestructuras del transporte marítimo-portuario ante el surgimiento de aquellas amenazas globales (como actos ilícitos, terrorismo internacional, ciberataques, accidentes intencionados o fortuitos, conflictos sociales y desastres naturales, esencialmente) que plantean nuevos escenarios de gestión de la seguridad, sean estos de carácter preventivo o, en cambio, de carácter reactivo. Tal circunstancia es, sin lugar a dudas, la motivante del marco normativo internacional, de obligado cumplimiento para cada uno de los Estados miembro, implementado por la Organización Marítima Internacional (OMI), así como de la estrategia europea de seguridad y protección portuaria, impulsada por la Comisión y materializada mediante la Directiva 2005/65/CE del Parlamento Europeo y del Consejo, de 26 de octubre de 2005, sobre mejora de la protección portuaria.

Este rasgo, como podrá deducirse en el estudio, presenta una especial significación en el tratamiento de la gobernanza en escenarios convergentes de las políticas públicas de seguridad y protección portuaria.

b) La especificidad de los actores intervinientes en la implementación de este tipo de política pública. Esencialmente, nos referimos, aquí, a la importancia que tienen en el proceso de acción pública las Fuerzas y Cuerpos de Seguridad del Estado, así como las Policías portuarias y Agentes de vigilancia aduanera, al conjugar su peculiar idiosincrasia con el ejercicio legítimo de autoridad pública y la interacción con su entorno.

c) El subespacio de las políticas públicas de seguridad y protección portuaria presenta una estrecha conexión con el subespacio de protección de infraestructuras críticas, así como con el subespacio de gestión de crisis y emergencias, con independencia de la propia idiosincrasia de cada uno de ellos. Por ello, el tratamiento específico de las vulnerabilidades será el que acote la interacción entre los referidos subespacios de las políticas públicas de seguridad. No obstante, la seguridad y protección portuaria presenta una marcada orientación de carácter preventivo, regulador, estratégico, evalua- 
tivo y resiliente que, conjugada con la eficacia y eficiencia de su gestión, permite potenciar tanto la coordinación como la cooperación en dicha interacción.

d) Con todo, es preciso señalar que el negativo impacto político, social y económico derivado de la inoperatividad de las infraestructuras portuarias, consecuencia de los actos ilícitos que potencian el riesgo y sus factores subyacentes, cuantificados en daños a personas, daños materiales y fallos del sistema, constituirá otro de los factores de especial significación en el proceso de diseño e implementación de las políticas públicas objeto de análisis en este artículo.

e) Finalmente, el rendimiento y la eficacia de las políticas públicas de seguridad y protección portuaria, así como los instrumentos provenientes de este subespacio, deben responder a una serie de elementos basales y de gestión que permitan la medición de la eficacia y eficiencia de este tipo de política pública. Entre ellos pueden identificarse: la zonificación de la infraestructura portuaria en función de los riesgos derivados de cada tipo de actividad; el diseño y ejecución continúa del análisis de riesgos como herramienta singular para la toma de decisiones en materia de seguridad y protección portuaria; el diseño, implementación y ejecución de los distintos planes de seguridad y protección portuaria; el análisis y evaluación de los resultados de la política implementada, así como de los impactos generados por los planes de seguridad y protección diseñados al efecto, y la definición e implementación de los criterios y mecanismos de interacción externa e interna.

La conjunción de todos y cada uno de los elementos configuradores, así como de los rasgos significativos de las políticas de seguridad y protección portuaria propuestos, supone la proyección de un subespacio de acción gubernamental diferenciado y configurado a través de microcircuitos sistémicos que contienen elementos de legitimación o deslegitimación en función de los estilos planteados para la consecución de los resultados e impactos finales. Ello implica, entonces, que se trata de un tipo de política pública que contiene un alto componente institucional, ya que su gestión tiene un marcado carácter demostrativo tanto de legitimidad como de autoridad gubernamental.

\section{LAS POLÍTICAS PÚBLICAS DE SEGURIDAD Y PROTECCIÓN PORTUARIA EN ESCENARIOS DE GOBERNANZA CONVERGENTE}

El actual escenario de gestión de las políticas públicas de seguridad y protección portuaria, condicionado y estimulado conjuntamente por los factores y los rasgos señalados en el apartado anterior, concentra nuevos marcos conceptuales y de pensamiento gerencial concordantes con los riesgos, las estrategias y los modelos de gestión pública de las infraestructuras portuarias españolas, así como con las demandas de servicios provenientes de su entorno.

Por ello, desde que la seguridad y protección portuaria fue considerada como un subespacio diferenciado de las políticas públicas de seguridad, recuérdese primera década del siglo XXI, se entendió que su eficacia y su eficiencia, así como su éxito o fracaso, dependería en mayor medida del abandono de viejos postulados de gestión y de la asunción, por lo tanto, de complejos paradigmas de cooperación y coordinación de carácter multiactoral y multinivel.

De este modo, nos encontramos ante un subespacio que esboza un peculiar escenario de gobernanza pública, en el que convergen factores de carácter supranacional que prevalecen sobre la concepción clásica de la acción reticular entre actores, así como de la corresponsabilidad, en el diseño e implementación de este tipo de política pública. Es decir, que no solo determina la acción reticular y las altas cotas de corresponsabilidad entre actores heterogéneos, así como la interacción de estos con su entorno, sino que, además, en dicho escenario ocupa, y debe ocupar, un lugar preeminente el acervo normativo nacional y supranacional en materia de seguridad y protección portuaria, al concebirse como el elemento armónico de «buen gobierno» en el ámbito de la Administración portuaria.

Tomando como referencia distintas nociones de gobernanza ${ }^{3}$, podemos conceptualizar a esta como el conjunto de actores multinivel público-privados que interactúan entre sí, y de modo reticular, con unos objetivos comunes y capacidad de decisión -ambos- en el curso de acción político-administrativa.

El tratamiento del concepto de gobernanza propuesto está inspirado, fundamentalmente, en las aportaciones realizadas al mismo por: Natera (2005), Aguilar Villanueva (2005 y 2014), Prats (2005) y Vanaclocha (2013). 
No obstante, partiendo de esta definición es preciso señalar, sin ánimo exhaustivo, los siguientes rasgos fundamentales del concepto. Desde la perspectiva de la gestión pública, es una extensión del concepto de la Nueva Gestión Pública (NGP). Su acción va más allá del carácter exclusivo de las instituciones y actores políticos en la gestión de los asuntos públicos, generando la existencia de una pluralidad de centros de decisión de carácter multicéntrico y multinivel. Identifica a múltiples actores -públicos y privados- que interactúan en red con la facultad de intervención en la gestión de los asuntos públicos. Y, finalmente, genera interdependencias de carácter multiactoral, fraguando mecanismos de cooperación y participación en la toma de decisiones y en la asunción de responsabilidades, así como liderazgos de tipo transaccional dentro de la red.

La gobernanza entonces, y como en un principio podría entenderse, no es algo fruto del azar, al tratarse de «procesos que no son aleatorios, discrecionales, irrestrictos, sino que están estructurados por las instituciones y por el conocimiento, poseyendo una estructura institucional y cognoscitiva» (Aguilar Villanueva, 2014: 17). Esta perspectiva permite poner el acento sobre la importancia de la cultura en las relaciones existentes entre las instituciones y la sociedad, así como en la transparencia, y en el acceso de la participación de los ciudadanos en la gestión de los asuntos públicos.

De este modo, la estratégica a seguir en el planteamiento reticular del subespacio de las políticas de seguridad y protección portuaria debe distinguir dos dimensiones diferenciadas: la dimensión derivada de las relaciones internas de la red, que será la encargada de la puesta en valor de las distintas relaciones, el nivel de confianza y las alianzas entre actores que faciliten la consecución de los objetivos prefijados; y la dimensión relativa a la gestión de la estructura reticular del modelo para adaptarla a las distintas estrategias que son impulsadas a través de un factor clave como es el liderazgo (Natera, 2005: 18).

Por tanto, no es difícil intuir que las políticas de seguridad y protección portuaria son proclives a constituir un espacio-red de gobernanza por excelencia. En esta red se identifican, por una parte, las estructuras portuarias y servicios de carácter territorial, así como factores y estructuras de carácter multinivel y transnacional; y por otra, en el ámbito estrictamente privado, las empresas concesionarias de las instalaciones portuarias en las que su actividad está relacionada de algún modo con la seguridad y protección. Se trata, por tanto, de un conjunto de actores heterogéneos que, en consonancia con la convergencia derivada del acervo normativo, comparten la corresponsabilidad de garantizar la seguridad y protección del entramado portuario, al generar su colapso o destrucción un potencial impacto económico y social en unas infraestructuras catalogadas como críticas.

Es entonces la significación que el entramado normativo transnacional tiene en las políticas públicas de seguridad y protección portuaria la que nos lleva a plantear en este artículo la gobernanza convergente como categoría de análisis. Recuérdese que esta responde a la influencia que el entramado normativo internacional, de obligado cumplimiento para las instituciones político-administrativas de cada Estado, tiene sobre el marco de interacción reticular entre actores multinivel, de carácter público y privado, que comparten objetivos comunes y capacidad de decisión en el curso de acción pública de las políticas objeto de análisis.

Así, tras la celebración en 1974 de la Conferencia de los Gobiernos Contratantes del Convenio Internacional para la Seguridad de la Vida Humana en el Mar (Convenio SOLAS), se aprobaron las resoluciones relativas a las enmiendas de su Anexo-capítulos V y XI2-, así como las relativas al Código Internacional para la Protección de los Buques e Instalaciones Portuarias (PBIP). Sin embargo, dichas resoluciones fueron

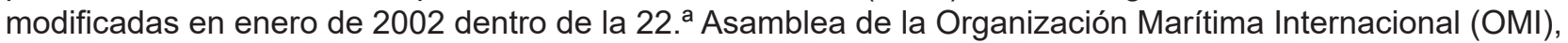
acordando de forma unánime la toma de medidas concurrentes a la mejora de la protección de buques y de las instalaciones portuarias ${ }^{4}$, al radicar sus aspectos motivadores en los actos terroristas y de piratería global que durante la primera década del presente siglo afectaron sobremanera a la actividad marítima internacional ${ }^{5}$.

El contexto de los actuales escenarios de gestión de la seguridad y protección portuaria emerge, por tanto, de las enunciadas normas transnacionales ${ }^{6}$. No obstante, el ámbito al que deben adaptarse todas

\footnotetext{
4 Dentro de las medidas de mejora de la protección de buques e instalaciones portuarias, el Código identifica como actos delictivos, los relativos a terrorismo, contrabando, robos de carga, polizones, inmigración ilegal, piraterías, así como los denominados daños colaterales causados por incendios y explosiones en buques e instalaciones portuarias, independientemente de cuál sea su origen.

5 Puede destacarse entre estos sucesos, los atentados terroristas del World Trade Center de Nueva York del 11 de septiembre de 2001 y de Madrid del 11 de marzo de 2004, así como los ataques derivados de la piratería sufridos por los buques Limburg y Han Wei en 2002.

6 La Organización Marítima Internacional (OMI) aprobó en 1974 el Convenio Internacional para la Seguridad de la Vida Humana en el Mar (SOLAS), que en su anexo incluye las normas y requisitos reguladores de la seguridad de buques y personas. El citado Con-
} 
las infraestructuras portuarias internacionales se complementa, a nivel europeo, con el Reglamento (CE) 725/2004 relativo a la mejora de la protección de los buques y las instalaciones portuarias -norma del ámbito comunitario derivada de las enmiendas al Anexo del Convenio SOLAS, que afecta a los capítulos $\mathrm{V}$ y XI $2-\mathrm{y}$ con la Directiva 2005/65/ $\mathrm{CE}^{7}$ de protección portuaria.

El marco normativo descrito a escala europea considera el nivel de protección del puerto en su conjunto y no solo a las instalaciones portuarias -ámbito de aplicación de las normas implementadas por la Organización Marítima Internacional-. En consonancia con ello, y acotando el análisis al caso español, son las Autoridades Portuarias como Administración portuaria de carácter descentralizado y de conformidad con el acervo normativo estatal y autonómico en materia de protección civil y emergencias, la facultada para elaborar los Planes de Emergencia Interior (PEI), integrándose los mismos en el acervo de las Ordenanzas portuarias.

Como extensión lógica de lo anterior, y con la finalidad de gestar una protección preventiva eficaz de actos antisociales y terroristas, es de obligado cumplimiento para cada una de las Autoridades Portuarias realizar el diseño y la implementación de un Plan específico para la protección de buques, pasajeros y mercancías, que de igual modo formará parte de las citadas Ordenanzas. Desde esta perspectiva, la normativa reguladora que establece las medidas para la mejora de la protección de los puertos y del transporte marítimo ${ }^{8}$, obliga a la Administración portuaria al diseño de un Plan de Protección Portuaria (PPP) que, con carácter inclusivo, debe imbricar los protocolos de coordinación con el Plan de Emergencia Interior (PEI), así como con el Plan Interior de Contingencias del Puerto (PICP).

En todo caso, la Autoridad Portuaria será el órgano competente para la supervisión y control del acervo normativo derivado del sistema de seguridad marítimo-portuario -incluyendo los derivados de la protección contra actos antisociales y terroristas-. Asimismo, será la responsable, en lo concerniente a las instalaciones portuarias, de decidir el ámbito de aplicación de la normativa internacional reguladora de seguridad y protección portuaria.

Todo ello, supone la necesidad de diseñar e implementar herramientas de gestión, análisis y evaluación de los riesgos ${ }^{9}$ que afectan de modo empírico a las infraestructuras portuarias, máxime si a estas las enmarcamos en la esfera de las infraestructuras catalogadas como críticas, ya que su actividad es indispensable y no permite soluciones alternativas, por lo que su perturbación o destrucción tendría un grave impacto -social y económico- sobre los servicios esenciales del país (Carmona, 2013: 108) ${ }^{10}$. En tal caso, los riesgos derivados de los actos ilícitos y antisociales inciden sobre las infraestructuras portuarias, al ser consideradas y definidas, dado su desarrollo e impacto económico supranacional, como uno de los elementos esenciales de la cadena internacional del transporte de mercancías y personas.

Consecuencia de ello es que el marco internacional de cooperación y coordinación de la seguridad portuaria precisa para su análisis la detección previa de las amenazas supranacionales y, por tanto, la implantación de un conjunto de protocolos operativos de prevención sobre los riesgos derivados de la propia idiosincrasia de las infraestructuras portuarias españolas. Desde esta perspectiva, no resulta difícil advertir cómo el modelo de gestión reticular de las políticas de seguridad y protección portuaria basa su integridad

venio ha sido enmendado en diversas ocasiones, desde su aprobación por la Conferencia diplomática de los gobiernos Contratantes, para adaptarlo a las nuevas necesidades de seguridad marítima-portuaria internacional. Para ello, en el año 2001 se puso en marcha el Comité de Seguridad Marítima con la facultad de proponer las distintas enmiendas al anexo del Convenio SOLAS, así como de la aprobación del Código Internacional para la Protección de Buques e Instalaciones Portuarias (Código PBIP-ISPS).

7 En el ámbito europeo, la normativa emanada de la Organización Marítima Internacional (OMI) ha sido complementada por la Directiva 2005/65/CE del Parlamento Europeo y del Consejo, de 26 de octubre de 2005, sobre mejora de la protección portuaria (DOUE de 25 de noviembre), cuyo objeto de aplicación es el conjunto del recinto portuario, extendido a determinadas zonas anexas que puedan constituir un riesgo en la actividad portuaria. De este modo, los factores de riesgo se afrontan mediante el establecimiento de estándares de gestión que incluyen la implantación de determinados planes de seguridad y protección, el uso de los medios para el control y la prevención, así como el sistema de respuesta ante contingencias singulares. Es preciso señalar en este sentido, que la trasposición de esta directiva al acervo normativo español tuvo lugar en el año 2008; si bien su aplicación efectiva al conjunto de puertos de interés general no se produjo hasta el año 2011.

8 Real Decreto 1617/2007, de 7 de diciembre, por el que se establecen medidas de mejora para la protección de los puertos y del trasporte marítimo (BOE de 20 de diciembre).

9 La gestión, análisis y evaluación de los riesgos portuarios se presenta como un elemento omnipresente en la dinámica comprendida por las políticas de seguridad y protección portuaria, al radicar su finalidad en la consecución óptima y eficaz de los medios en relación con las necesidades.

10 El autor clasifica los conceptos basales de estructura del sistema de protección de infraestructuras críticas en función de lo recogido en la Ley $8 / 2011$, de 28 de abril, por la que se establecen las medidas para la protección de las infraestructuras críticas (BOE de 29 de abril). 
en dos aspectos esenciales: la seguridad ${ }^{11}$-safety- y la protección ${ }^{12}$-security-. Por ello, la convergencia entre ambos elementos incide directamente en el grado de interdependencia, así como en la integración de los distintos planes y programas parciales de seguridad portuaria, en un plan de ámbito superior como es el Plan de Protección de Instalaciones Portuarias (PPIP).

Así pues, la complejidad y los rasgos funcionales implícitos en las políticas públicas de seguridad y protección portuaria son los factores que permiten identificarlas como espacios proclives a la gobernanza; principalmente en la actualidad, donde los modelos de gestión de la seguridad marítimo-portuaria están siendo fruto de una constante revisión derivada de la identificación de nuevos riesgos y amenazas. En este sentido, la lógica inspirada por la denominada gobernanza convergente permite modular el análisis y la redefinición de problemas para encontrar soluciones eficaces y eficientes en un subespacio de especial significación de la acción pública. Pero, además, ayuda a comprenderlo en toda su extensión, al permitir su análisis reconocer las claves de la cooperación, de la colaboración y de las relaciones de confianza entre los actores públicos y privados intervinientes, así como la importancia que tiene en el escenario de la seguridad y protección portuaria el acervo regulador de carácter transnacional.

\section{CONCLUSIONES}

Los factores configuradores, así como los rasgos característicos de la seguridad y protección portuaria, permiten profundizar en su complejo marco de acción, en la construcción de nuevos paradigmas de gestión y en la implementación de instrumentos y estrategias diferenciados. Ello supone que nos encontramos ante un subespacio de especial significación de las políticas públicas de seguridad.

A lo largo del artículo se han podido mostrar diversos factores y rasgos característicos que permiten constatar que el subespacio que comportan las políticas de seguridad y protección portuaria, entendido como la expresión de la acción gubernamental conducente a la prestación pública de la seguridad por la Administración portuaria española de titularidad estatal, se muestra de modo complejo e indeterminado.

En este sentido, es preciso destacar que (1) Tanto el diseño como su implementación provienen de la iniciativa supranacional para el análisis, la gestión y la prevención de las vulnerabilidades y riesgos ante el surgimiento de amenazas globales que son susceptibles de vulnerar el normal funcionamiento de las infraestructuras portuarias. (2) En consonancia con lo anterior, dichas amenazas plantean nuevos escenarios de gestión que irrumpen en paralelo con la emergencia y consolidación de las políticas públicas de seguridad y protección portuaria. (3) El subespacio objeto de análisis presenta una estrecha conexión con otros subespacios característicos de las políticas públicas de seguridad como son en esencia el de las infraestructuras críticas y el de la gestión de crisis y emergencias. (4) Un hecho singular en el proceso de diseño e implementación de este tipo de política pública es el negativo impacto político, económico y social que deriva de la inoperatividad de las infraestructuras del transporte portuario. (5) El rendimiento y la eficacia de estas políticas públicas, así como los instrumentos imbricados en el subespacio de la acción pública, deben responder a una serie de elementos basales y de gestión que faciliten la medición y evaluación de su eficacia y eficiencia. Y, (6) como último rasgo característico, se infiere una especial singularidad de los actores intervinientes en la implementación de las políticas públicas de seguridad y protección portuaria (Fuerzas y Cuerpos de Seguridad del Estado, Policías Portuarias y Agentes de Vigilancia Aduanera, esencialmente), al conjugar su peculiar idiosincrasia con el ejercicio legítimo de autoridad pública y la interacción con el entorno.

No obstante, el análisis de las políticas de seguridad y protección portuaria demuestra que no solo se construyen como resultado de la combinación de paradigmas, de modelos organizativos y de sistemas de respuesta a las demandas tradicionales, sino que existe una razón que explica la armonización de dichas políticas a escala nacional e internacional: la transposición normativa transnacional de obligado cumplimiento que configura la planificación y la gestión de la seguridad en las infraestructuras portuarias. Los factores de diseño que homogeneizan las políticas públicas objeto de análisis son evidentes, pero, como ha podido deducirse, se relativizan por la diferenciación que deriva de categorizar a la gobernanza como convergente.

11 La seguridad (safety) tiene como misión la reducción de los incidentes y de las posibles causas de emergencia, al ejecutar una mecánica de acontecimientos previsibles y cuantificables en función de los factores y amenazas del entorno, así como de los protocolos de prevención y del factor humano.

12 Sin embargo, la protección (security) es un concepto integrado y ratificado por un espectro de voluntades, propósitos y objetivos derivados de cada uno de los planes propios e interdependientes de seguridad portuaria. 
Por consiguiente, la gestión reticular convergente de las políticas públicas de seguridad y protección portuaria deriva de un escenario de gobernanza diferenciado, al presumirse la influencia del acervo normativo transnacional. En este sentido ha podido deducirse que tanto sus instrumentos como sus estrategias quedan enmarcados por las distintas disposiciones recogidas por el Código Internacional para la Protección de Buques e Instalaciones Portuarias (PBIP), en función de lo dispuesto por la Organización Marítima Internacional $(\mathrm{OMI})$ para toda infraestructura portuaria española con tráficos internacionales. Sin embargo, es preciso advertir que esta perspectiva reticular precisa la implementación de distintos instrumentos de cooperación y de colaboración, así como de relaciones de confianza entre los diversos actores que participan en el proceso, para la consecución de niveles aceptados de eficacia y eficiencia en el ámbito de la seguridad y protección portuaria.

\section{REFERENCIAS BIBLIOGRÁFICAS Y DOCUMENTALES}

AGUILAR VILLANUEVA, L. F. (1992): La hechura de las políticas públicas. México D. F.: Porrúa.

- 2011: Gobernanza y gestión pública. México D. F.: Fondo de Cultura Económica.

- 2014: "Las dimensiones y los niveles de la gobernanza". Cuadernos de Gobierno y Administración Pública, 1(1): 11-36. DOI: 10.5209/rev_CGAP.2014.v1.n1.45156 (consulta: 14 abril 2017).

BAÑÓN, R. y JIMÉNEZ, S. (2010): La nueva gestión pública. Madrid: TQM.

BAUMAN, Z. (2011): Daños colaterales. Desigualdades sociales en la era global. México D. F.: Fondo de cultura económica.

BECK, U. (1992): Risk Society. Londres: Polity Press.

- 1998: World Risk Society. Cambridge: Polity Press.

BOIN, A., HART, P., STERN, E. y SUNDELIUS, B. (2005): The Politics of Crisis Management: Public Leadership under Pressure. Cambridge: Cambridge University Press. DOI: $10.1017 / 9781316339756$.

BROOKS, M. R. (2004): "The Governance Structure of Ports". Review of Netwoks Economics, 3(3): 168-183. DOI: 10.2202/1446-9022.1049 (consulta: 1 octubre 2017).

CARMONA, C. (2013): "Marco conceptual del sistema español de infraestructuras críticas", en VANACLOCHA, F. J. dir.: Marco legal y de gestión de la protección de infraestructuras críticas en España. Madrid: McGraw-Hill.

- 2014: "Modernización y modelo policial español: Estudio de caso de la Unidad de Policía Adscrita a la Junta de Andalucía", en BAÑÓN, R. y TAMBOLEO, R., dirs.: La modernización de la gestión y los asuntos públicos. Madrid: Universidad Complutense.

- 2015: "La modulación de las organizaciones policiales ante una ciudadanía exigente: una perspectiva sistémica", en BAÑÓN, R. y TAMBOLEO, R., dirs.: Ideas para la gobernanza. Madrid: Fragua.

DEL NERO, E. (2008): "Planificación de los puertos estatales y la ordenación del territorio". Revista de Obras públicas, 3491: 39-64. Disponible en Web: <http://ropdigital.ciccp.es/pdf/restringido/2008/2008_septiembre_3491_03.pdf> (consulta: 30 septiembre 2017).

DEZALAY, Y. y GARTH, B. (2002): "Legitimating the New Legal Orthodoxy”, en DEZALAY, Y. y GARTH, B. eds.: Global Prescription. The Production, Exportation, and Importation of a New Legal Orthodoxy. Ann Arbor: University of Michigan Press.

DREZNER, D. (2005): "Globalization, Harmonization, and Competition: The Different Pathways to Policy Convergence”, Journal of European Public Policy, 12(5): 841-859. DOI: 10.1111/1475-6765.00012 (consulta: 2 abril 2017).

FLIGSTEIN, N. (2008): Euro-Clash. The EU, European Identity and the Future of Europe. Oxford: Oxford University Press.

FRAMBRY, K. (2012): Crisis, Disaster and Risk. Institutional Response and Emergence. Londres: Eurospan. DOI: $10.4324 / 9781315705354$.

GOMÀ, R. y SUBIRATS, J. (1999): Políticas públicas en España: contenidos, redes de actores y niveles de gobierno. Barcelona: Ariel.

GIDDENS, A. (1990): The Consequences of Modernity. Cambridge: Polity Press.

KASSIM, H. (2001): The National Co-ordination of EU Policy: The European Level; The Domestic Level. Oxford: Oxford University Press.

KINGDOM, J. W. (1995): Agendas, Alternatives and Public Policies. Nueva York: Harper Collins.

KNILL, C. y LEHMKUHL, D. (2002): "The national impact of European Union regulatory policy: Three Europeanization mechanisms". European Journal of Political Research, 41: 255-280. DOI: 10.1111/1475-6765.00012 (consulta: 27 marzo 2017).

KOOIMAN, J., ed., (1993): Modern Governance: New Government-Society Relations. Londres: Sage.

LINDBLOM, E. (1959): “The Science of 'Muddling Through'”. Public Administration Review, 19: 79-88. Disponible en web: <https://faculty.washington.edu/mccurdy/SciencePolicy/Lindblom\%20Muddling\%20Through.pdf> (consulta: 29 marzo 2017). 
LOSADA, R. (2005): "Buscando el riesgo aceptable: sobre los métodos de análisis de riesgo empleados en la elaboración de las políticas públicas”, Gestión y análisis de políticas públicas, 33-34: 27-50. DOI: 10.24965/gapp. vi33-34.390 (consulta: 2 octubre 2017).

LOWI, T. (1972): "Four Systems of Policy, Politics, and Choice". Public Administration Review, 32: 298-310. DOI: 10.2307/974990 (consulta: 29 marzo 2017).

MATHAN, R. E. (1995): "Synthesizing the Implementation Literature: The Ambiguity Conflict Model of Policy Implementation”. Journal of Public Administration Research and Theory, 5(2): 145-174. Disponible en web: <http:// orion.luc.edu/ rmatlan/pdf/1995SynthesizingthelmplementationLiterature.pdf> (consulta: 23 marzo 2017).

MENY, I. y THOENING, J. C. (1992): Las políticas públicas. Barcelona: Ariel.

METCALFE, L. (1993): “Public Management: From Imitation to Innovation”, en Kooiman, J. ed.: Modern Governance. Londres: Sage.

NATERA PERAL, A. (2005): "El factor liderazgo en la gestión de redes de gobernanza", en NATERA, A. y VANACLOCHA, F. J. dirs.: Los liderazgos en el mercado político y la gestión pública. Madrid: BOE.

- 2005: "La gobernanza como modo emergente de gobierno y gestión pública". Gestión y Análisis de Políticas Públicas, 33-34: 53-65. DOI: 10.24965/gapp.vi33-34.391 (consulta: 14 abril 2017).

OLÍAS DE LIMA, B., coord. (2001): La nueva gestión pública. Madrid: Prentice Hall.

POWELL, W. y DIMAGGIO, P. J. (1991): "The Iron Cage Revisited: Institutional Isomorphism and Collective Rationality in Organizational Fields", en POWELL, W. y DIMAGGIO, P., eds.: The New Institutionalism in Organizational Analysis. Chicago: University of Chicago Press.

PRATS, J. (2005): De la burocracia al management, del management a la gobernanza. Madrid: INAP.

RUANO, J. M. (2014): "La política local de seguridad en las grandes ciudades. El caso de la transformación de la policía municipal de Madrid”. Cuadernos de Gobierno y Administración Pública, 1(2): 139-157. DOI: http://dx.doi. org/10.5209/rev_CGAP.2014.v1.n2.47539 (consulta: 3 junio 2017).

SIMON, H. (1947): Administrative Behavior. Londres: Macmillan.

SUBIRATS, J. (1989): Análisis de políticas públicas y eficacia de la Administración. Madrid: INAP.

SUBIRATS, J. y GOMÁ, R. (2001): "Las políticas públicas”, en ALCÁNTARA, M. y MARTÍNEZ, A., eds.: Política y gobierno en España. Valencia: Tirant lo Blanch.

SUBIRATS, J., KNOEPFEL, P., LARRUE, C. y VARONE, F. (2011): Análisis y gestión de las políticas públicas. Barcelona: Ariel.

TALAVERA, F. (2014): “El sistema nacional de protección civil”. Cuadernos de estrategia, 165: 19-68. Disponible en web: <http://www.ieee.es/publicaciones-new/cuadernos-de-estrategia/2014/Cuaderno_165.html> (consulta: 22 abril 2017).

TAMAYO, M. (1997): "El análisis de las políticas públicas", en BAÑÓN, R. y CARRILLO, E., eds.: La nueva Administración pública. Madrid: Alianza.

VANACLOCHA, F. J. (2009): "Gobernanza en las políticas públicas de seguridad. Una nueva perspectiva sobre el lugar de la seguridad privada”, en Fundación Policía Española, coord.: Seguridad pública-Seguridad privada. ¿Dilema o concurrencia? Madrid: FPE.

- 2013: "La protección de infraestructuras críticas como espacio emergente de políticas públicas y gobernanza", en VANACLOCHA, F. J., dir.: Marco legal y de gestión de la protección de infraestructuras críticas en España. Madrid: McGraw-Hill.

VELÁZQUEZ, F. J. (2009): "La seguridad privada en el sistema de seguridad pública”, en Fundación Policía Española, coord.: Seguridad pública-Seguridad privada. ¿Dilema o concurrencia? Madrid: FPE.

WALLACE, H. y WALLACE, W., eds. (2000): Policy-making in the European Union. Oxford: Oxford University Press. 